\title{
Sketch-Based Virtual Human Modelling and Animation
}

\author{
Chen Mao, Sheng Feng Qin, and David Wright \\ School of Engineering and Design, Brunel University, Uxbridge, UB8 3PH, UK \\ \{Chen.Mao, Sheng.Feng.Qin, David.Wright\}@brunel.ac.uk
}

\begin{abstract}
Animated virtual humans created by skilled artists play a remarkable role in today's public entertainment. However, ordinary users are still treated as audiences due to the lack of appropriate expertise, equipment, and computer skills. We developed a new method and a novel sketching interface, which enable anyone who can draw to "sketch-out" 3D virtual humans and animation. We devised a "Stick Figure $\rightarrow$ Fleshing-out $\rightarrow$ Skin Mapping" graphical pipeline, which decomposes the complexity of figure drawing and considerably boosts the modelling and animation efficiency. We developed a gesture-based method for $3 \mathrm{D}$ pose reconstruction from $2 \mathrm{D}$ stick figure drawings. We investigated a "Creative Model-based Method", which performs a human perception process to transfer users' 2D freehand sketches into 3D human bodies of various body sizes, shapes and fat distributions. Our current system supports character animation in various forms including articulated figure animation, 3D mesh model animation, and 2D contour/NPR animation with personalised drawing styles. Moreover, this interface also supports sketch-based crowd animation and 2D storyboarding of 3D multiple character interactions. A preliminary user study was conducted to support the overall system design. Our system has been formally tested by various users on Tablet PC. After minimal training, even a beginner can create vivid virtual humans and animate them within minutes.
\end{abstract}

Keywords: Sketching interface, Virtual human modelling and animation, Storyboarding, User study.

\section{Sketch-Based Human Modelling and Animation Pipeline}

\subsection{Intuitive Graphics Pipeline}

Natural figure sketches are featured by foreshortening, contour over-tracing, body part overlapping, shading and shadow, etc. To decompose the complexity of direct 3D modelling and animation from fully rendered sketches, we designed a "Stick Figure $[1] \rightarrow$ Fleshing-out $\rightarrow$ Skin Mapping" pipeline (see Fig. 1). This is inspired by the drawing sequence recommended by many sketch books and tutorials. Meanwhile, in principle, it echoes the modelling and animation pipeline in commercial packages (i.e. Maya, 3D Studio Max). Regarding our current design, functionalities at different levels were gained for different users. Thus they can choose to make simple stick figures, create delicate 3D surface models, or explore further to animate these sketchgenerated creatures. Moreover, models can be exported to commercial packages at any level, to be refined by their powerful function kits. 


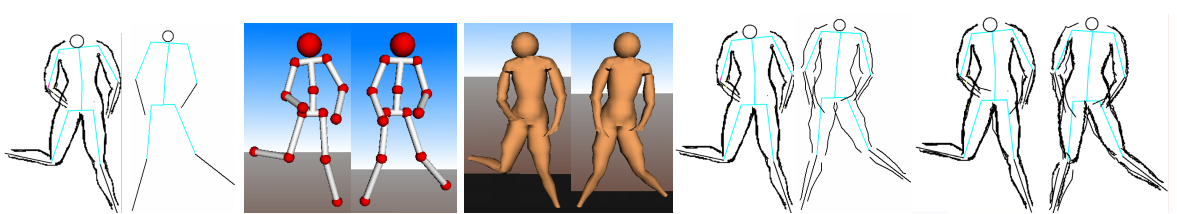

Fig. 1. Users first draw stick figure key frames to define a specific motion. Then, they can "flesh-out" any existing stick figure with body profiles to portray an imaginative character. The system can automatically "perceive" the body size (skeleton proportion) and shape (body profile and fat distribution) from the sketched figure, and transfer it into a 3D virtual character. The resulting 3D skin surface can be mapped onto each of the posed stick figure key frames, which can be further interpolated as the final 2D and 3D character animation.

\subsection{Preliminary User Study}

A preliminary user study (including Questionnaire and Sketching Observation) was conducted to explore design questions, identify users' needs, and obtain a "true story" of figure sketching. 60 questionnaires were collected and processed; and 14 sketching observations were conducted with 14 users (including artists, designers, animators, graduate students, and researchers) in the pre-defined drawing scenarios. The study outcomes were adopted to develop a natural and supportive sketching interface.

\subsection{Sketch-Based 3D Stick Figure Modelling and Animation}

Maintaining the correct proportion and foreshortening is a common challenge in figure sketching for not only novice but also skilled artists. To help overcome this difficulty, we provide an on-line drawing assistance, which is based on the utilisation of template skeleton and the real-time body part recognition and length control. We developed a "Multi-layered Back-front Ambiguity Clarifier", which utilises figure perspective rendering gestures, human joint ROM (Range of Motion), and key frame coherence to identify user intended 3D poses from 2D stick figure drawings. Since a quick and imprecise sketching may accidentally generate physically impossible poses, we offer a "Figure Pose Checking and Auto-correction" routine to detect ill-posed body parts, highlight them, and give proper corrections based on human body joint ROM and balance. Once obtaining a series of 3D stick figure poses, user can interactively sketch-out motion paths and timing, and add their favoured sound/background to enhance the 3D virtual world. The resulting 3D animation can be automatically synthesised in VRML after a single user click.

\section{Fleshing-Out to Create Various Human Body Models}

\subsection{From 2D Raw Sketches to 3D Human Body Models}

Users can depict the visual appearance of a virtual character through "fleshing-out" a single stick figure with body profiles. Improved on [2], which enables sketch-based spherical object modelling, we investigated a "Creative Model-based Method", which performs a human perception process to model irregular/complicated human skin 
surfaces. Through this method, the system can perceive the body size (skeleton proportion) and shape (body fat distribution) of a sketched figure and transfer it into a plausible 3D counterpart model through continuous graphical comparisons and generic model morphing (see Fig. 2). We created a three-layered (skeleton-fat tissueskin) anatomical generic model, which can be transformed sequentially through rigid morphing, fatness morphing, and surface fitting to match the original 2D sketch. Moreover, we offer an auto-beautification function to regularise 3D asymmetrical bodies made by users' drawing imperfections. Users can interactively refine their 3D models by over-sketching 2D figure profiles. Modifications can be made at any time and on any key frame sketch to achieve the updated 3D model.

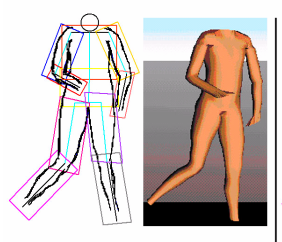

(a)

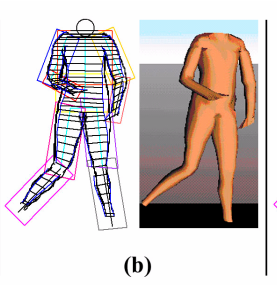

(b)

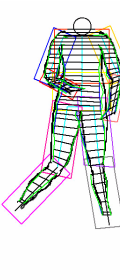

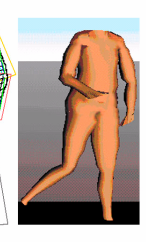

(c)

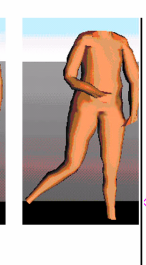

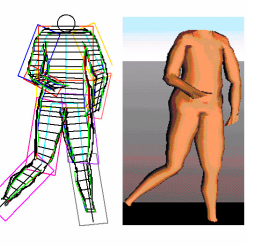

(d)

Fig. 2. (a) The input $2 \mathrm{D}$ freehand sketch and the $3 \mathrm{D}$ model after rigid morphing. (b) Graphical comparison to get the fat distribution measurements and the fatness morphed model (c) Graphical comparison to get the surface fitting measurements; the model with and without system auto-beautification (d) Overtracing body contour (right lower torso, left lower leg, and right leg) to modify an existing 3D surface model.

\subsection{Generic Model Acquisition and Specification}

Our generic model is reconstructed from cadaveric cross-section images from the Visible Human Project ${ }^{\circledR}$ of the National Library of Medicine. The preparation of this multi-layered anatomical model includes: Virtual Skeleton Registration, Skin Mesh Recovery, Template Fat Distribution Digitisation.

\section{Sketch-Based Multi-level Character Animation}

Our system supports character animation in multiple levels, including articulated figure animation, 3D mesh model animation, and 2D contour animation, and 2D personalized NPR animation (see Fig. 1). Following the "Stick Figure $\rightarrow$ Fleshingout $\rightarrow$ Skin Mapping" pipeline, a 3D virtual human animation is accomplished by wrapping the sketch-generated skin surface onto a series of posed stick figures, which can be further interpolated via VRML with the associated graphical motion definition. 2D NPR animation is generated by successively interpolating the extracted key figure contours (with the mapped fleshing-out contours) and playing their in-betweens on sketching interface. This approach is different from the traditional cel animation, and users do not need to render each key frame once a single key figure is fleshed-out. 


\section{Sketching-Out Crowd Animation and Character Interactions}

In our system, users can build their own 3D character and motion library, and animate a population of virtual humans through motion retargeting and a sketch-based actor allocation in 3D space [3]. Moreover, users are able to sketch-out character interactions in each story scene (by either stick or full figure drawing). The system can deliver an immediate 3D scenario, in which virtual actors are acting and interacting with each other.

\section{User Experiences}

This system has been tested by 10 different users (including artists, an animator, undergraduates and postgraduates, and a 12-year-old boy) through performance tests, sketching observation, and user interviews. After a short tutorial, users rapidly learned the modelling process and began to sketch-out their own virtual human animation on Tablet PC within minutes. Only 6.27 and 6.75 minutes were taken on average to create a 3-frame stick and full figure animation respectively. Through sketching observation and user interviews, our sketching interface is proved by users to be easy to learn and use, and enjoyable for fast 3D virtual human modelling and animation.

\section{Conclusion}

Human modelling and animation is a recognised challenge and labour-intensive task, which has been, until now, confined to the domain of professionals. This research draws on existing drawing skills of ordinary users to create and animate their own characters through 2D freehand sketching. Preliminary user study and formal user tests were conducted to ensure the usability and functionalities of this sketch-based modelling and animation interface.

\section{References}

1. Mao, C., Qin, S.F., Wright, D.K.: A Sketch-based Gesture Interface for Rough 3D Stick Figure Animation. In: Proc. of Eurographics Workshop on Sketch Based Interfaces and Modeling, pp. 175-183 (2005)

2. Igarashi, T., Matsuoka, S., Tanaka, H.: Teddy: A Sketching Interface for 3D Freeform Design. In: Proc. SIGGRAPH ‘99, pp. 409-416 (1999)

3. Mao, C., Qin, S.F., Wright, D.K.: Sketching-out Virtual Humans: from 2D Storyboarding to Immediate 3D Character Animation. In: Proc.of ACM SIGCHI International Conference on Advances in Computer Entertainment Technology, Hollywood, Los Angeles (2006) 